\title{
A Combined SHM / IDA Method for Assessing Collapse Capacity and Risk in Subsequent Ground Motions
}

\author{
Jessica Fitzjohn ${ }^{\text {a }}$, Cong Zhou ${ }^{\mathrm{a}}$, J. Geoffrey Chase ${ }^{\mathrm{a}}$ \\ ${ }^{a}$ Department of Mechanical Engineering, University of Canterbury, Private Bag 4800
}

Christchurch 8140, New Zealand

\begin{abstract}
Prediction of building collapse due to significant seismic motion is a principle objective of earthquake engineers, particularly after a major seismic event when the structure is damaged and decisions may need to be made rapidly concerning the safe occupation of a building or surrounding areas. Traditional model-based pushover analyses are effective, but only if the structural properties are well understood, which is not the case after an event when that information is most useful. This paper combines Hysteresis Loop Analysis (HLA) structural health monitoring (SHM) and Incremental Dynamic Analysis (IDA) methods to identify and then analyse collapse capacity and probability of collapse for a specific structure, at any time, a range of earthquake excitations to ensure robustness. This nonlinear dynamic analysis enables constant updating of building performance predictions following a given and subsequent earthquake events, which can result in difficult to identify deterioration of structural components and their resulting capacity, all of which is far more difficult using static pushover analysis. The combined methods and analysis provide near real-time updating of the collapse fragility curves as events progress, thus quantifying the change of collapse probability or seismic induced losses very soon after an earthquake for decision-making. Thus, this combination of methods enables a novel, higher resolution analysis of risk that was not previously available. The methods are not computationally expensive and there is no requirement for a validated numerical model, thus providing a relatively simpler means of assessing collapse probability immediately post-event when such speed can provide better information for critical decision-making. Finally, the results also show a clear need to extend the area of SHM toward creating improved predictive models for analysis of subsequent events, where the Christchurch series of 2010-2011 had significant post-event aftershocks.
\end{abstract}

Keywords: Structural health monitoring; SHM, Incremental dynamic analysis; IDA; Collapse capacity; $P$-Delta, Hysteresis loop analysis 


\section{Introduction}

Increasing urbanisation worldwide has resulted in increased housing development, concentration, and resulting greater number of taller buildings [1], in both non-seismic and seismic zones. Seismic damage is thus a major risk with significant follow-on social and economic impacts. Structural health monitoring (SHM) arose from the need to accurately detect, localise and quantify damage after major seismic or environmental loads. Further, significant damage in a major event can be exacerbated in subsequent large aftershocks, increasing the risk to occupants, as well as the structure itself.

SHM thus addresses the immediate needs of responders and leadership. However, it does not provide a ready, quantified means of assessing, the ongoing safety of a structure or the, potentially now modified, risk of collapse. This outcome requires a model to be made from the SHM results and either existing building data or a reasonably accurate surrogate. Such a model would enable further linear and nonlinear analyses to assess the risk of collapse and thus enable more optimal decision making for occupants and owners, as well as decision makers presiding over a city with many such structures.

Collapse prediction under seismic loading is one of the principal objectives of earthquake engineering [2-4]. In particular, large lateral displacements in tall structures can lead to $P$-delta effects $[1,5] . P$-delta effects increase overturning moments, generating effectively negative post-yield stiffnesses, and thus increase the likelihood of global collapse. Degradation in strength and stiffness are also a related important consideration for buildings in seismic zones where inelastic response is common for major events, as inelastic response can degrade nominal and post-yield stiffness, generate consistent net added overturning moments, and thus increase the potential for collapse [2]. Hence, there is a need to know the structural stiffness 
degradation and inelastic net deformation to better estimate collapse risk. Figure 1 illustrates these effects $[6,7]$.

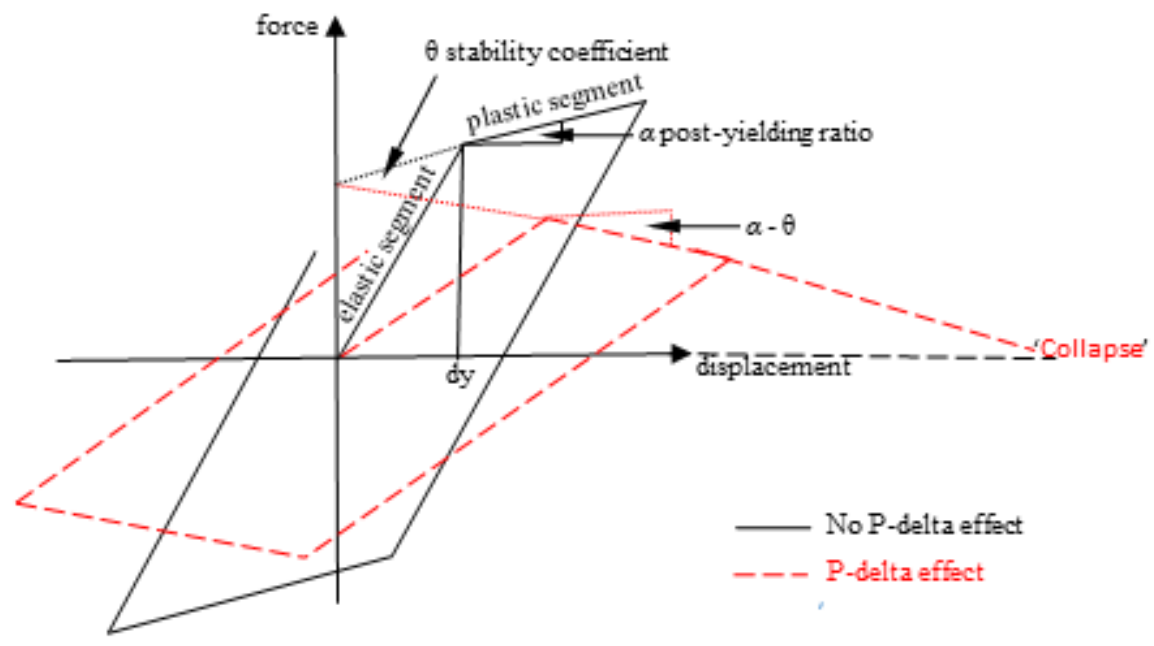

Figure 1. Schematic illustration of bilinear cyclic behaviour including P-Delta and nominal stiffness degradation effects, all of which together can result in an effective negative postyield stiffness.

The degree of stiffness degradation is dependent on structural characteristics, such as material properties, geometry and connection types [8]. For structures with stability coefficients leading to negative stiffness, degradation is less of a concern, as usually gravity effects resulting in inelastic deformation will lead to rapid onset of collapse [2]. Other studies demonstrate more significant effects of stiffness degradation for structures built on soft soil [9-12]. Hence, degradation and $P$-delta effects must be considered in monitoring structures and their behaviour to best estimate further risk after an event $[13,14]$. 
In addition, current SHM methods only assess damage states, but cannot predict future risk of collapse. More specifically, they can quantify damage in terms of stiffness changes, or more commonly, frequency changes, but cannot transform those results into quantified measures of risk of collapse or financial loss, which are necessary to aid decision making by responders and owners. The lack of trusted, accurate risk assessment of future damage and collapse led to significant disagreements about the level of damage and remaining lifetime of several structures in Christchurch, New Zealand after the events of 2010-2011, delaying repair and recovery [15]. On 22 February 2011, the already damaged CTV building collapsed, killing 115 of 1805 fatalities in this catastrophic event.

There is thus a need to link the results of SHM to prediction of further structural capacity, including $P$-delta effects, to enable better prediction of subsequent structural behaviour and thus risk. Incremental Dynamic Analysis (IDA) linked to SHM identified models offers a solution. IDA of practical structures is computationally demanding and, as a result, simplified methods approximating structures as single degree of freedom systems have been developed to provide approximations [4]. In addition, pushover analysis has been widely used to obtain the structural parameters for the simplified computational model required for implementing IDA $[2,4,6,16-20]$. However, static pushover analysis is based on the assumption structures primarily oscillate in the first mode during earthquakes, where a static response spectrum, rather than a set of ground accelerations, is used to capture dynamic performance [21]. Hence, this approach can lack resolution and lead to inaccuracy, and a computational model created from dynamic analysis is needed to provide better accuracy.

In particular, Adam and Jager predicted earthquake induced side-sway collapse of nondeteriorating moment resistant frame structures vulnerable to $P$-delta effects [22]. They utilised 
a similar collapse capacity assessment using IDA, but with static pushover analysis used to identify parameters leading to collapse. However, this study disregarded deterioration of structural components and thus their initial state assumed an undamaged initial structure, which may not be the case post-event [13].

The objective of this work is to combine SHM and IDA to extend SHM results from a damagemonitoring role into a more comprehensive tool for risk assessment of collapse and financial loss. A nonlinear hysteretic model can be created and updated based on the identified HLA SHM results after each major event, which thus significantly reduces the modelling uncertainty commonly seen in static push over analysis. In addition, the differences in dynamic seismic response to different events are also considered by using a wide range of earthquake records scaled for equal probability of occurrence. Finally, the implementation of IDA with an accurate computational model and this suite of earthquake records ensures accurate risk assessment of collapse and financial loss, extending SHM results from quantifying damage into quantifying risk and loss inputs for more optimal decision making.

This study is a proof of concept numerical analysis for dynamic IDA linked to a proven SHM method to provide potentially valuable risk assessment relatively immediately post-event. Hysteresis Loop Analysis (HLA) is an accurate, proven, real-time dynamic SHM method [13, 14, 23-27], providing realistic structural parameters for subsequent collapse prediction analyses. This study is the first to utilise HLA, or to our knowledge any SHM method, in combination with nonlinear IDA to identify collapse capacity and probability of collapse. 


\section{Methods}

The proof of concept system is modelled as a single degree of freedom (SDOF) structure using an elastic-plastic bilinear hysteric model, and analyses cases with and without initial or prior stiffness degradation. Such a bilinear model has been widely used to represent the basic inelastic system with P-delta effect $[2,6,17,28,29]$, where the yielding displacement $d y$ divides the response into an elastic segment and a plastic segment as shown in Figure 1. The HLA SHM method [25] is used to determine the current damaged or undamaged structural parameters of the system. IDA methods [18] using a suite of earthquake records with incremented intensity are utilized with nonlinear dynamic analyses to generate collapse capacity spectra quantifying the probability of collapse for the SHM identified post-event structure under this range of input seismic loading.

Figure 2 outlines the overall analysis process. To implement HLA and IDA, ground acceleration, structural acceleration and/or displacement need to be measured during the earthquake. Structural mass can be evaluated based structural configuration and material properties of the building. 


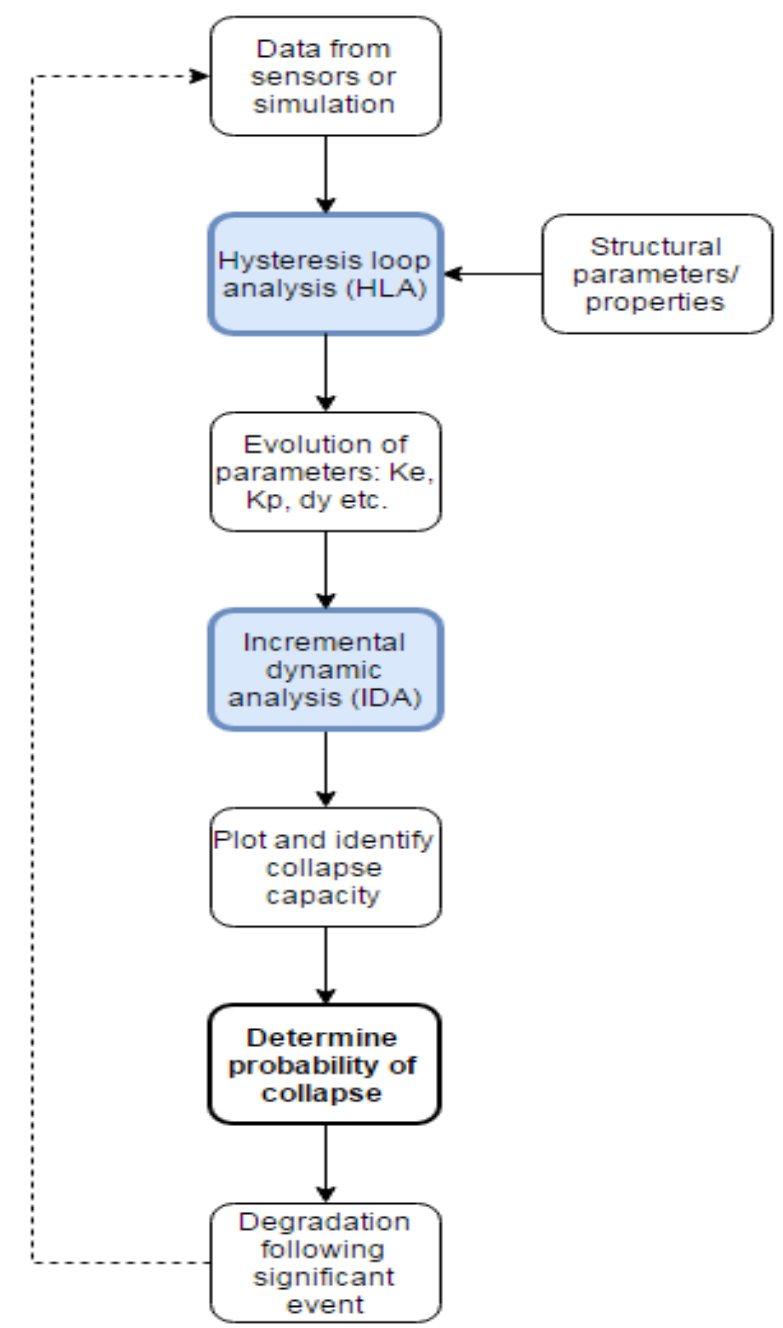

Figure 2. Flowchart of the overall method, where the HLA and IDA sections are highlighted

\subsection{Hysteresis Loop Analysis (HLA)}

Hysteresis Loop Analysis (HLA) [25] was used to identify building parameters including stability and hardening coefficient, elastic and plastic stiffness, and yielding displacement. It removes the need for the typically used static pushover analysis, which requires assuming first mode collapse, a structural model, and structural parameters [3], where assuming a structural model in SHM can lead to errors if it is not accurate [14]. The HLA method extracts significant half cycles of seismic or ambient response using sensor data. Elastic and plastic stiffness values are identified using statistical hypothesis testing from the hysteretic force-displacement response. Yielding displacement is thus found from the maximum deflection prior to plastic 
deformation, and need not be based on assumed structural parameter values in a model, reducing uncertainty and error.

In particular, the equation of motion of a single degree of freedom (SDOF) is defined:

$$
m \ddot{x}+c \dot{x}+F(x)=-m \ddot{x}_{g}
$$

where $x, \dot{x}$ and $\ddot{x}$ are the displacement, velocity and acceleration of the SDOF system; $m$ is the mass of the system; $F(x)$ is the restoring force of the hysteresis system; and $\ddot{x}_{g}$ is the ground acceleration; $c$ is the viscous damping coefficient with equation. Thus, the restoring force can be calculated:

$$
F(x)=-m \ddot{x}_{g}-m \ddot{x}-c \dot{x}
$$

The hysteresis loop can then be plotted using restoring force $F(x)$ and the displacement $x$ for this SDOF system, as shown in Figure 3. The hysteresis loop can then divided by the turning point where the displacement is local maximum or minimum. Finally, the breakpoint can be found using hypothesis test $[25,26]$ to calculate the slope values for each elastic and plastic segments.
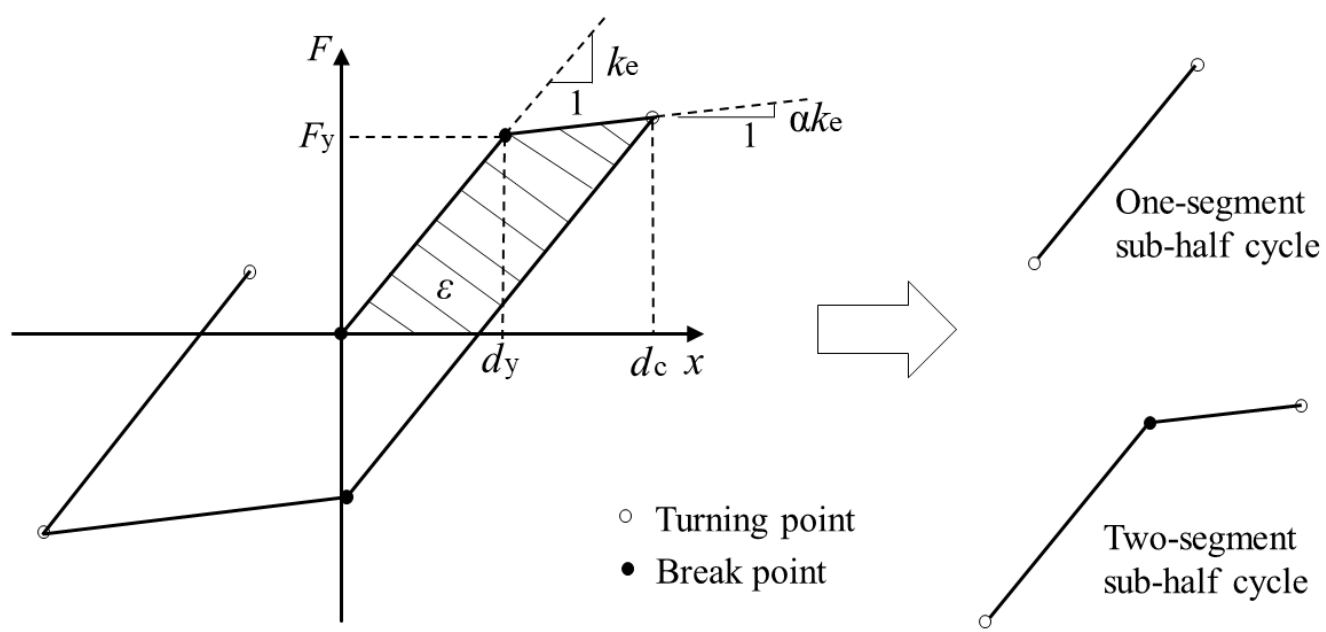

Figure 3. Example of how HLA divides half cycles and calculate stiffness $k e$ and $k p$. 
The stability coefficient is a measure of the sensitivity to P-delta effect. It is the ratio of the second overturning moment due to structural weight to the bending moment due to the lateral seismic force [5], which is thus defined:

$$
\theta=\frac{P \Delta}{F h}=\frac{P}{K h}=\frac{m g}{K h}
$$

where $\theta$ is the stability and hardening coefficient and a large value of $\theta$ is more likely to yield a negative stiffness and collapse. $K$ is the initial structural stiffness, assumed or identified using HLA, excluding $P$-delta effects, $P$ is the weight $(\mathrm{kN})$ of the building, $h$ is the height of the building.

The equations used to determine the stiffness values are defined:

$$
\begin{aligned}
& K_{e}=(1-\theta) K \\
& K_{p}=(\alpha-\theta) K
\end{aligned}
$$

where $\alpha$ is the stability and hardening coefficient, and $K_{e}$ and $K_{p}$ are the values for elastic and plastic stiffness from the HLA identification.

Thus, the stability coefficient $\theta$ can be rewritten as a function of identified $K_{e}$ and $K_{p}$ values from the HLA SHM method, and defined:

$$
\theta=\frac{m g}{K_{e} h+m g}=\frac{m g \alpha}{K_{P} h+m g}
$$

\subsection{Incremental Dynamic Analysis (IDA)}

Incremental Dynamic Analysis (IDA) is a parametric analysis method used to more thoroughly estimate building performance under seismic loading to quantify the relationship between seismic capacity and demand, potentially leading to evaluation of financial risk $[3,16,18,30$, 31]. This relationship is found by subjecting a structural model to varying ground motion records scaling the intensity of the records in increments until, in this case, collapse is predicted 
[3]. This analysis results in multiple curves of any intensity measure (IM), such as peak ground acceleration, plotted against any engineering demand parameter (EDP), such as maximum deflection or drift. $[6,7,16]$. This plot quantifies the largest earthquake intensity at which a structure maintains, in this case, stability for each particular ground motion and is used to determine the global collapse capacity of a specific structure $[2,18]$.

There is no unique definition to characterize the intensity of an earthquake record $[2,18,32]$. An accepted method is to use the normalized spectral acceleration damped at $\xi=5 \%$ at the structures fundamental period $\left(S_{a}\left(T_{1}, 5 \%\right)\right)$ as a fraction of the product of acceleration of gravity, $g$, and the base shear coefficient, $\gamma$. In this study, spectral acceleration at the structures first mode period has been used as the intensity measure, as it has been found to be an efficient, structure specific intensity measure compared to peak ground acceleration or velocity, which are not site specific [32], where the site can have significant impact on response [12].

The result of the IDA is thus highly dependent on the earthquake record used, as the variability of different ground motions leads to different collapse capacities [30]. Thus, the analysis presented is performed for a range of $n$ ground motion inputs [33], providing a range of ground motion spectra and thus adding robustness to the results.

The maximum intensity measure where a small increase of intensity lead to dynamics instability is defined as the collapse capacity for a specific earthquake [34]. The classification of the collapse capacity for multiple earthquake events, $C C_{i}$, is defined:

$$
C C_{i}=\frac{S_{a, i}}{g \gamma} \quad \text { for } i=1, \ldots, n
$$

Where $g$ is gravitational constant and $\gamma$ is defined: 


$$
\gamma=\frac{f_{y}}{m g}
$$

Where $m$ is the total mass of the structure and $f_{y}$ is defined:

$$
f_{y}=d_{y} K
$$

Where $d_{y}$ is the yielding displacement and $K$ is the initial structural stiffness, assumed or identified using HLA or another SHM method.

This analysis utilized peak ductility, $\mu$, as the damage measure or EDP, which is defined:

$$
\mu=\frac{d_{\max }}{d_{y}}
$$

Where $d_{\max }$ is the maximum displacement response, and $d_{y}$ is the yielding displacement, which can be found from the response data from a prior event.

Collapse occurs when the plastic segment of the hysteresis loop in Figure 1 intersects the $x$ axis at a non-zero value. The increments in seismic input were thus refined accordingly to best represent the likely ductility range of a real structure for each earthquake to capture the intensity at collapse with good resolution, rather than relying on a fixed set of increments. The probability of collapse for the specific structure is then determined by fitting a log-normal distribution curve to the collapse capacity values, $C C_{i}$, for each earthquake to assess the magnitude causing collapse and its relative likelihood of occurrence.

\subsection{Degradation Analysis}

Stiffness degradation was incorporated in a second analysis based on the total energy dissipation for each cycle and calculated from the total area enclosed by the hysteresis loop for each half cycle. A stiffness degradation parameter, $\eta$, was defined: 


$$
\eta=1+\delta_{\eta} \epsilon
$$

where $\delta_{\eta}$ is the stiffness degradation constant, and $\epsilon$ is the energy dissipation for half cycles including plastic deformation. Therefore, the changing stiffness $K_{\eta}(t)$ can be defined:

$$
K_{\eta}(t)=\frac{1}{\eta} \times K
$$

This method allows continuous updating of the stiffness values throughout the analysis if degradation due to prior events is to be included.

HLA can identify stiffness degradation $K_{\eta}(t)$ over time, which thus offers an update of the collapse probability after each event. In addition, the degradation coefficient $\delta_{\eta}$ could also be calculated using Equations (11)-(12) once $K_{\eta}(t)$ is identified from HLA. In particular, the dissipated energy $\epsilon$ can be calculated by the accumulated plastic deformation in the bilinear model $[26,35,36]$, as shown in Figure 3. Energy dissipation could also be calculated using hysteretic displacement and identified stiffness degradation in other more complex hysteretic models $[37,38]$.

Note that degradation, as defined in Equations 11-12, can also be defined to mimic a known degradation measured during SHM for a given building or class of buildings. This approach can thus be used to mimic a structure during a prior event or to apply an assumed degradation to a similar structure in evaluating the potentially increased risk for a forthcoming event. 


\section{Case Study/ Proof of Concept Analysis}

This methodology is used in a proof of concept case study to determine the probability of collapse for a 7-storey steel moment resisting frame (SMRF) structure with known properties, as defined in Table 1 [39]. The initial stability coefficient, $\theta$, is calculated to be 0.1014 using Equation (6). The effect of stiffness degradation is included, as variation of $\theta, \alpha$ and $T_{0}$ would change the collapse capacity [7].

Table 1. Structural properties of steel MRF structure for analysis

\begin{tabular}{|c|c|c|}
\hline Total mass $(m)$ & 4 & $\mathrm{kN} \mathrm{s} / \mathrm{mm}$ \\
\hline Initial stiffness $(K)$ without P- $\Delta$ effect & 157.9 & $\mathrm{kN} / \mathrm{mm}$ \\
\hline Initial period $\left(T_{0}\right)$ & 1 & $\mathrm{~s}$ \\
\hline Height $(h)$ & $24.5^{*}$ & $\mathrm{~m}$ \\
\hline Hardening coefficient $(\alpha)$ & 0.02 & \\
\hline Yielding displacement $\left(d_{y}\right)$ & 24.85 & $\mathrm{~mm}$ \\
\hline
\end{tabular}

*The height was calculated assuming $3.5 \mathrm{~m}$ height per storey.

The structural response of the modelled structure subjected to an earthquake excitation is first simulated with added $10 \%$ RMS noise using the Newmark- $\beta$ integration method with a sampling frequency of $1000 \mathrm{~Hz}$ for the acceleration and a sampling frequency of $1 \mathrm{~Hz}$ for the displacement, representing realistic sensor measurements, sensor noise, and sampling rates [14, 25]. The HLA method is then applied to the modelled response with added noise to identify the structural parameters for input to the IDA procedure, thus simulating the overall SHM of a "real" structure with known behaviours in this case, followed by IDA using the identified structural parameters to assess the risk of collapse. 
The structural model is a typical SMRF building in California [39]. Therefore, a suite of 20 earthquake records in Table 2 representative of ordinary earthquakes recorded in California having a probability of exceedance of $10 \%$ in 50 years are selected to capture the typical range of structural response in the building areas[20, 33]. Given the identified structural parameters, the IDA procedure can then be performed using the selected 20 records to provide an accurate estimation of seismic demand. The IDA curves and the probability of collapse for the modelled structure are assessed for each specific ground motion from the 20 earthquake events. From this curve the exact intensity of collapse and its likelihood of occurrence are calculated.

There are thus results obtained using the HLA method and then subsequent IDA analysis. Equally, there are ground truth, direct simulation results to provide the "truth" for comparison. These results are compared for each event and on average to assess the performance of these two methods in combination, and thus their potential for use in real-time monitoring and decision making after an event.

These analyses are all run including $P$-Delta effects. They are run without including stiffness degradation first. Subsequently, stiffness degradation is included in a repeat analysis, using Equations 11-12. These analyses demonstrate both the added impact of stiffness degradation on eventual subsequent collapse, and the ability of the overall method presented to incorporate this added effect.

In each case, the results lead to a probability of collapse capacity, $C C_{i}$, for each event. These results are compared between simulated ground truth from the proof of concept case study, and the same value as determined via the combination of HLA structural identification and subsequent IDA analysis using those identified structural parameters from the SHM analysis. 
The comparison thus quantifies the potential of mixing proven SHM and IDA methodologies to rapidly provide collapse risk assessments right after a major event. 
Table 2. Earthquake records used for analysis [33]

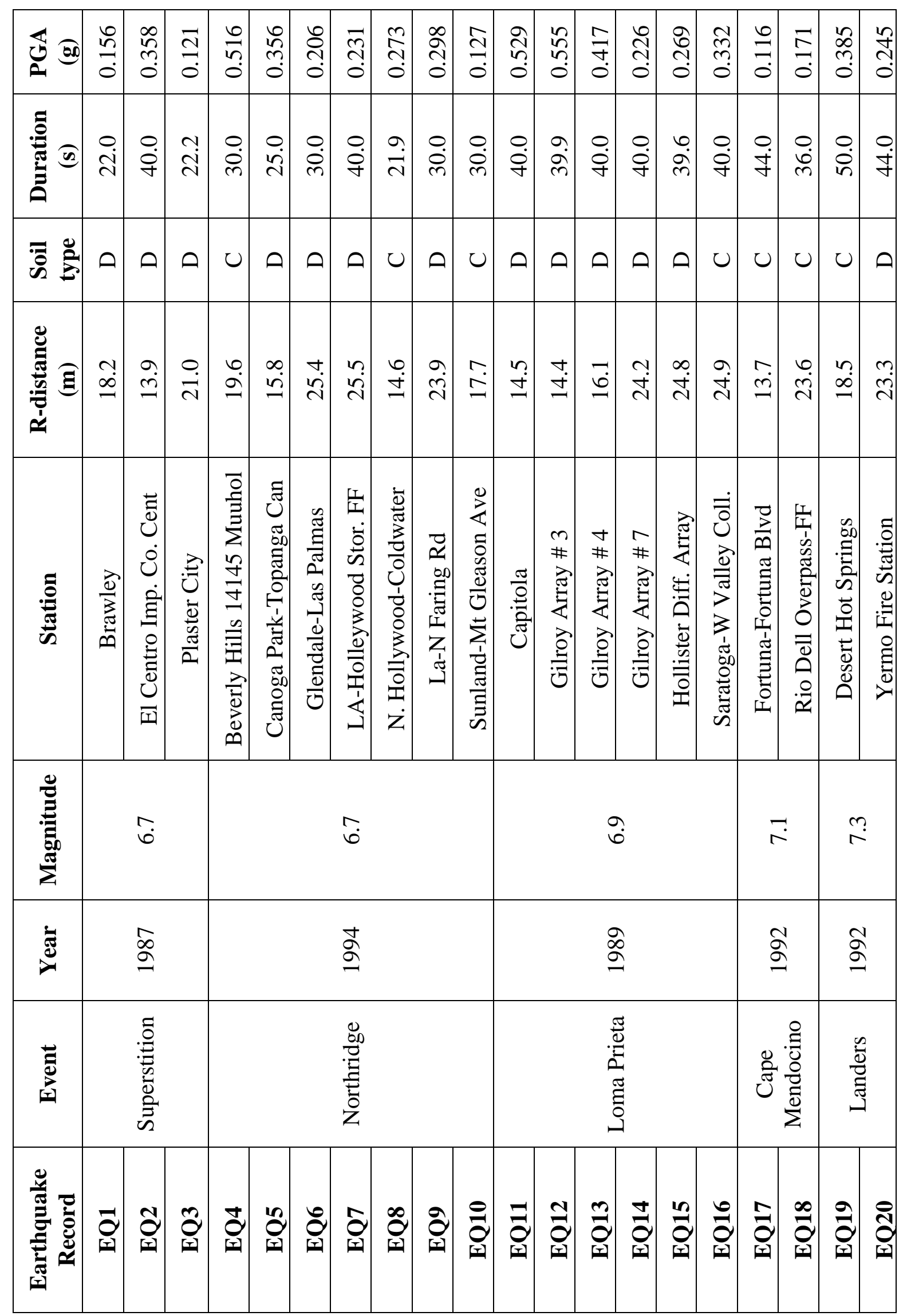




\section{Results and Discussion}

\subsection{Structural parameter identification}

The mean values of HLA identified structural parameters and resulting stability analysis for the 20 different earthquake events are compared with the ground truth results from direct numerical simulation in Table 3. The values identified are sufficiently close to the numerical model ground truth, despite the 10\% RMS added noise, matching prior analytical and experimental results [13, 14, 25-27]. HLA identified structural parameters for each earthquake, including the collapse capacity found using IDA, are shown in Table 4.

Values for plastic stiffness and yielding displacement were excluded where ground motions were not large enough to cause significant plastic deformation or when the structure was rapidly driven to collapse not allowing a sufficient number of plastic deformation cycles. Collapse capacity in all cases was identified through scaled magnitude of the ground motion until collapse. The IDA curves generated for incremented magnitude for each earthquake until collapse are shown in Figure 4. It shows the effects of different earthquake ground motions on the specified proof of concept structure. Additional lines showing the median and $16^{\text {th }}$ and $84^{\text {th }}$ percentile $(* /$ of 1 multiplicative standard deviation [40]) of these lognormal distributed values are also plotted in Figure 4.

Table 3. Mean values from analysis compared with simulation inputs

\begin{tabular}{|c|c|c|c|c|}
\hline & $\begin{array}{c}\text { Elastic stiffness } \\
\boldsymbol{K}_{\boldsymbol{e}}(\mathbf{N} / \mathbf{m})\end{array}$ & $\begin{array}{c}\text { Plastic stiffness } \\
\boldsymbol{K}_{\boldsymbol{p}} \mathbf{( N / m )}\end{array}$ & $\begin{array}{c}\text { Stability } \\
\text { coefficient } \theta\end{array}$ & $\begin{array}{c}\text { Yielding } \\
\text { displacement } \boldsymbol{d}_{\boldsymbol{y}}(\mathbf{m})\end{array}$ \\
\hline $\begin{array}{c}\text { Mean HLA } \\
\text { Identified Values }\end{array}$ & $1.42 \mathrm{E}+07$ & $-1.21 \mathrm{E}+06$ & 0.1017 & 0.0244 \\
\hline $\begin{array}{c}\text { Known Ground } \\
\text { Truth Simulation }\end{array}$ & $1.42 \mathrm{E}+07$ & $-1.29 \mathrm{E}+06$ & 0.1014 & 0.0249 \\
\hline
\end{tabular}


Table 4. Elastic \& plastic stiffness, stability coefficient, yielding displacement, peak ground acceleration and collapse capacity for 7-storey SMRF structure under 20 ground motion excitations

\begin{tabular}{|c|c|c|c|c|c|c|}
\hline EQ & $\begin{array}{c}\text { Elastic stiffness, } \mathbf{K}_{\mathrm{e}} \\
(\mathbf{N} / \mathbf{m})\end{array}$ & $\begin{array}{c}\text { Plastic stiffness, } K_{p} \\
(\mathbf{N} / \mathbf{m})\end{array}$ & Stability coefficient & $\begin{array}{c}\text { Yielding } \\
\text { displacement (m) } \\
\end{array}$ & $\begin{array}{c}\text { Peak ground } \\
\text { Acceleration (g) }\end{array}$ & Collapse Capacity \\
\hline 1 & $1.48 \mathrm{E}+07$ & $-1.22 \mathrm{E}+06$ & 0.10147 & 0.0236 & 0.123 & 23.56 \\
\hline 2 & $1.33 \mathrm{E}+07$ & - & 0.10782 & - & 0.360 & 4.71 \\
\hline 3 & $1.42 \mathrm{E}+07$ & - & 0.10139 & - & 0.122 & 48.22 \\
\hline 4 & $1.41 \mathrm{E}+07$ & $-1.16 \mathrm{E}+06$ & 0.10178 & 0.0247 & 0.519 & 14.62 \\
\hline 5 & $1.42 \mathrm{E}+07$ & $-1.14 \mathrm{E}+06$ & 0.10147 & 0.0274 & 0.357 & 7.59 \\
\hline 6 & $1.42 \mathrm{E}+07$ & - & 0.10132 & - & 0.207 & 53.58 \\
\hline 7 & $1.42 \mathrm{E}+07$ & $-1.21 \mathrm{E}+06$ & 0.10134 & 0.0247 & 0.233 & 13.77 \\
\hline 8 & $1.43 \mathrm{E}+07$ & $-1.27 \mathrm{E}+06$ & 0.10103 & 0.0245 & 0.285 & 11.65 \\
\hline 9 & $1.42 \mathrm{E}+07$ & - & 0.10148 & - & 0.190 & 48.54 \\
\hline 10 & $1.42 \mathrm{E}+07$ & $-1.24 \mathrm{E}+06$ & 0.10132 & 0.0241 & 0.127 & 17.60 \\
\hline 11 & $1.42 \mathrm{E}+07$ & $-1.15 \mathrm{E}+06$ & 0.10113 & 0.0226 & 0.453 & 18.01 \\
\hline 12 & $1.42 \mathrm{E}+07$ & $-1.22 \mathrm{E}+06$ & 0.10147 & 0.0223 & 0.540 & 12.41 \\
\hline 13 & $1.39 \mathrm{E}+07$ & - & 0.10321 & - & 0.305 & 11.91 \\
\hline 14 & $1.42 \mathrm{E}+07$ & - & 0.10143 & - & 0.227 & 27.52 \\
\hline 15 & $1.42 \mathrm{E}+07$ & $-1.12 \mathrm{E}+06$ & 0.10112 & 0.0248 & 0.212 & 7.93 \\
\hline 16 & $1.42 \mathrm{E}+07$ & $-1.22 \mathrm{E}+06$ & 0.10141 & 0.0251 & 0.049 & 4.85 \\
\hline 17 & $1.43 \mathrm{E}+07$ & $-1.32 \mathrm{E}+06$ & 0.10051 & 0.0248 & 0.054 & 7.23 \\
\hline 18 & $1.42 \mathrm{E}+07$ & $-1.23 \mathrm{E}+06$ & 0.10116 & 0.0248 & 0.245 & 17.71 \\
\hline 19 & $1.42 \mathrm{E}+07$ & $-1.19 \mathrm{E}+06$ & 0.10137 & 0.0249 & 0.172 & 14.27 \\
\hline $20^{2}$ & $1.42 \mathrm{E}+07$ & $-1.22 \mathrm{E}+06$ & 0.10102 & 0.0226 & 0.085 & 9.45 \\
\hline
\end{tabular}




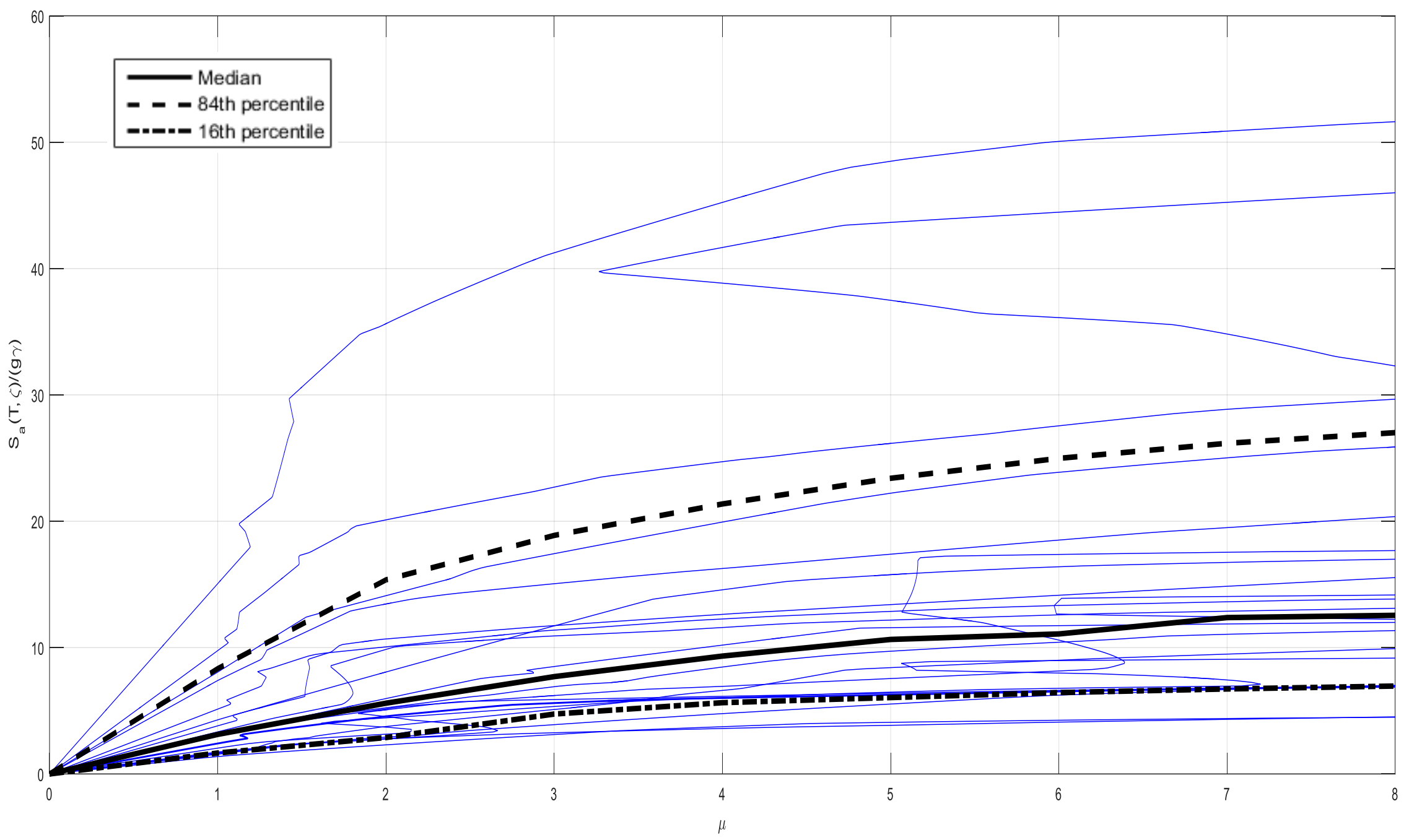

Figure 4. IDA curves for 20 ground motions applied to 7-storey SMRF structure with a fundamental period of 1.0s with the calculated median (solid), $16^{\text {th }}$ and $84^{\text {th }}$ (dashed) percentile lines included to show the overall distribution better. 
Table 5 indicates the median $\left(50^{\text {th }}\right), 84^{\text {th }}$ and $16^{\text {th }}$ percentile values of overall collapse capacity $\left(C C_{i}\right)$ over all 20 events, which is 1 multiplicative standard deviation in each direction from the median of the lognormal distributed results. Most earthquake ground motions lead to a softening structural response, with gradual degradation towards collapse. Some earthquakes lead to more severe hardening and non-monotomic structural behaviour, which has been previously reported [18]. This variation in results illustrates the varying demand structures are subject to during seismic excitation depending on the type and characterisation of the specific ground motion input [30], and further validates the use of multiple events to generate a robust risk profile.

Table 5. Median, 84th and 16th percentile values for collapse capacity, $C C_{i}$

\begin{tabular}{|c|c|}
\hline Median & 12.55 \\
\hline $84^{\text {th }}$ percentile & 27.01 \\
\hline $16^{\text {th }}$ percentile & 6.97 \\
\hline
\end{tabular}

The $C C_{i}$ value for a specific ground motion corresponds to the intensity measure (IM) $S_{a} / g \gamma$ when the story drift goes to infinity or dynamic side-sway instability of the structure is reached for a given ground motion. Thus, the $C C_{i}$ value varies over events due to the random nature of ground motions, which is denoted as aleatory uncertainty [20]. Hence, the median value in Table 5 may be considered as the representative $C C_{i}$ for this particular structure and the given set of 20 different events, while the $16^{\text {th }}$ and $84^{\text {th }}$ percentile of the $C C_{i}$ values characterizes their distribution statistically [6].

\subsection{Probability of collapse}

The consequent probability of collapse under seismic excitation for the 7-storey structure is shown in Figure 5 as a function of the collapse capacity value, $C C_{i}$. This result is generated based on the collapse capacity of the structure found for all 20 different earthquake excitations from the IDA. Figure 5 shows that the probability of collapse of a particular structure can be reasonably estimated by a log-normal distribution, as also noted in the presentation of the results in Tables 4-5 and Figure 4. 


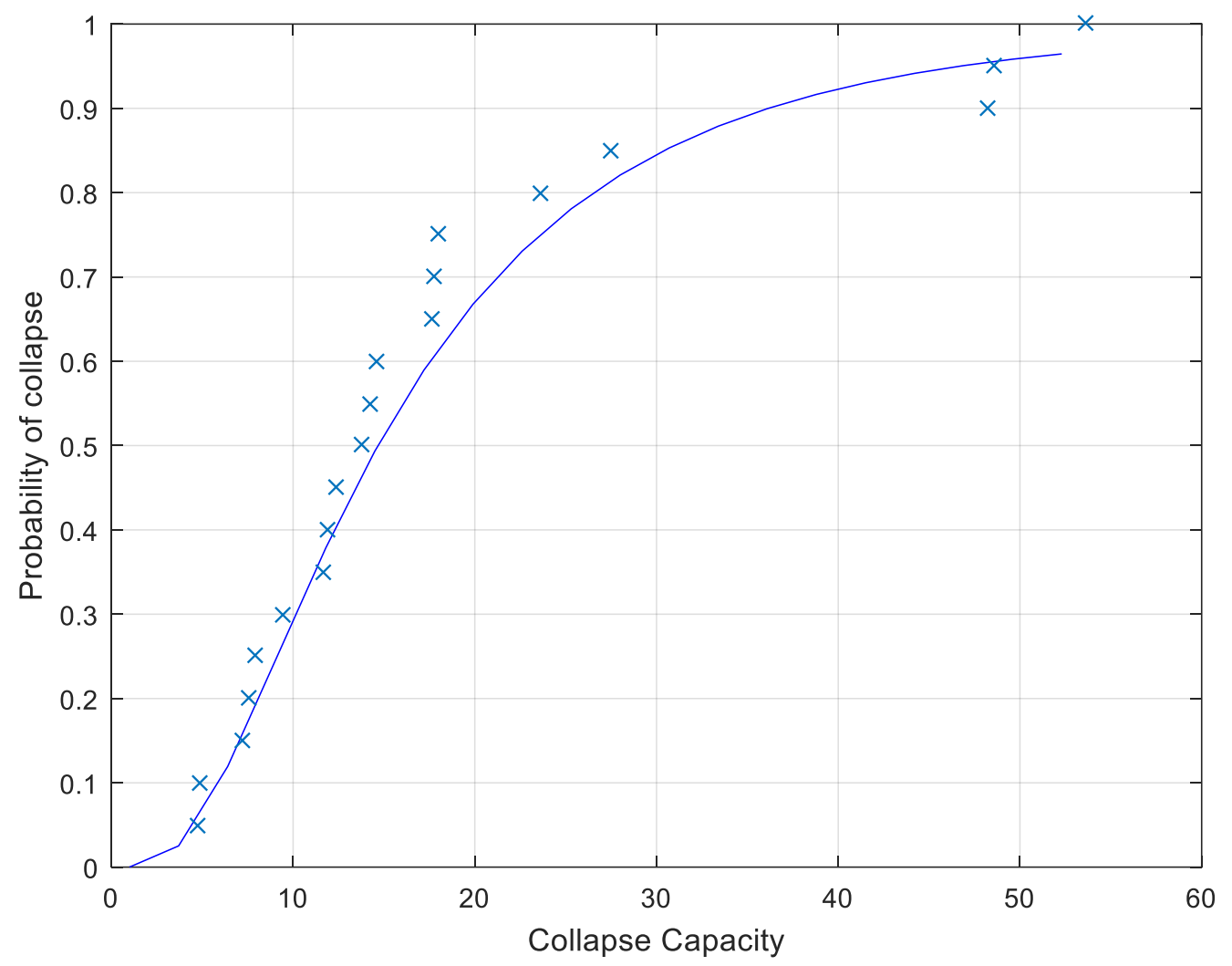

Figure 5. Counted collapse fragility curve of the 20 earthquakes and analytical approximation of collapse probability based on log-normal distribution

\subsection{Stiffness degradation analysis}

Further analysis including stiffness degradation per Equations 11-12 assesses the impact of stiffness degradation, where the structure is assumed to be further damaged during a subsequent event from the initial event used to find the HLA identified structural parameters. In general, it would be assumed that a structure would thus collapse more easily and at a lower intensity as a result of continuing structural degradation. As a result, the risk is expected to increase with the curve of Figure 5 moving left. However, this analysis quantifies the amount of this change, and thus whether its inclusion is necessary given the reasonable, assumed degradation profile defined in Equations 11-12.

Seismic motion from EQ19 in Table 2 is used to illustrate the extent of degradation in both elastic and plastic stiffness in a subsequent event, and to illustrate its impact on the hysteresis loops used in the HLA method. Figure 6 shows the hysteretic response of the structure to EQ19 with (left) and without 
(right) stiffness degradation, where $P$-delta effects are included in both cases. The changes are qualitatively reasonable for such a large event. Finally, Figure 7 compares the changes of elastic stiffness over time between the HLA identified values and the simulated true values found using Equations 11-12 in the case study structure, validating the ability of the HLA method to provide accurate estimation of structural parameters for the IDA procedure.
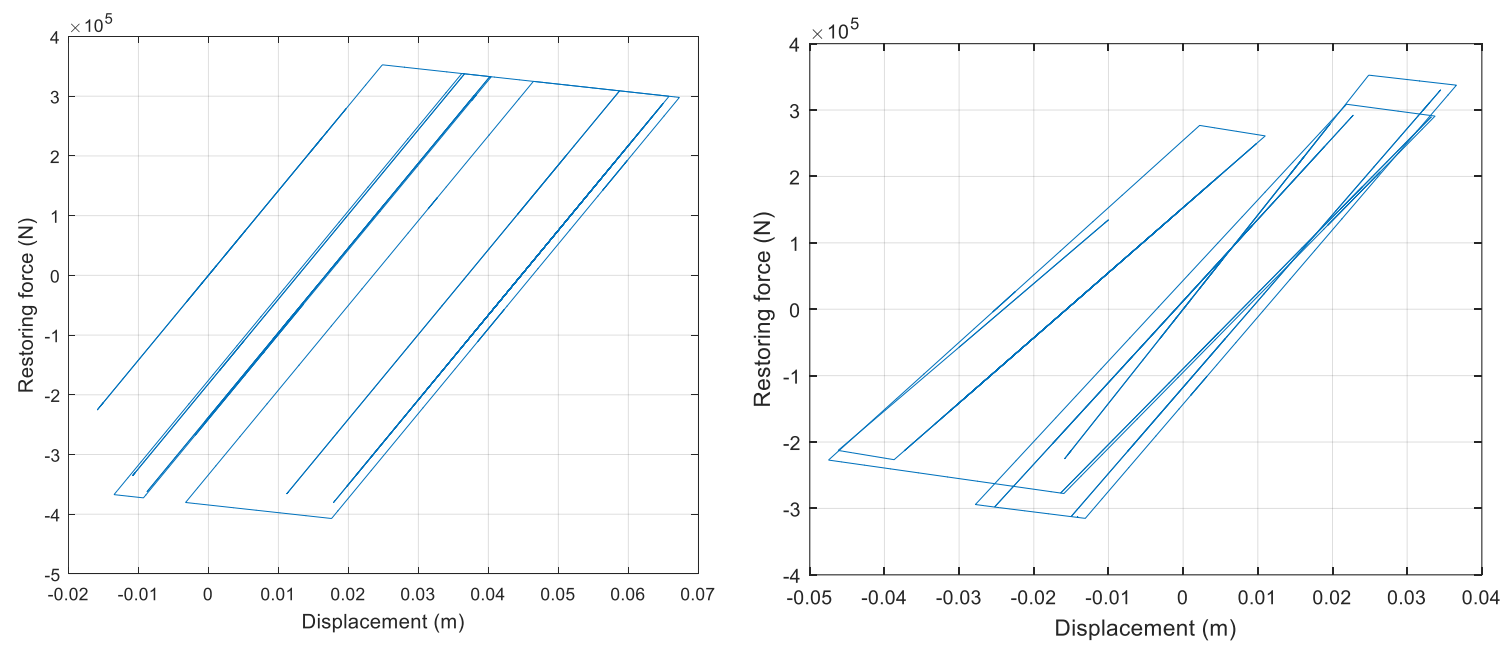

Figure 6. Earthquake 19 hysteresis loop excluding stiffness degradation but including $P$-delta effects (left); and including stiffness degradation and $P$-delta effects (right)

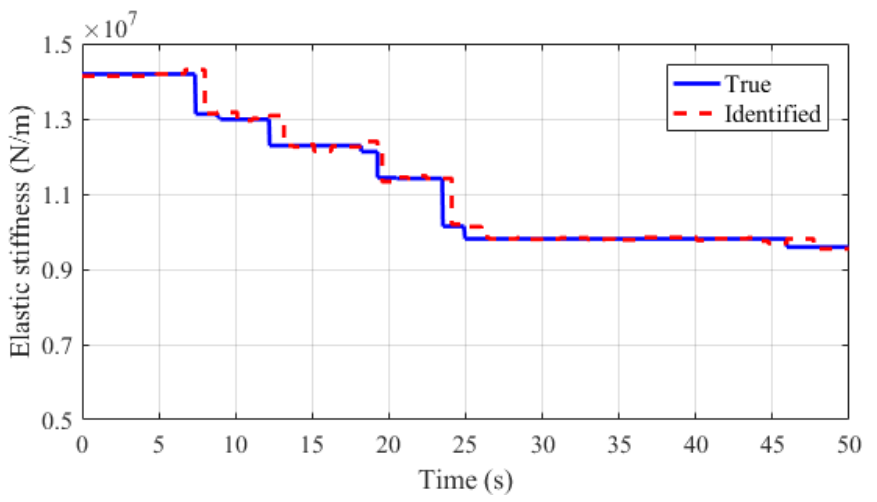

Figure 7.Comparision of stiffness degradation between the identified and true values.

While Figures 6-7 show an example, IDA was implemented across all 20 earthquake events with the updated structural parameters from HLA identification used for each subsequent event. The probability of collapse is then re-evaluated considering the continuing degradation of stiffness combined with $P$ - 
delta effects. Figure 8 compares the new collapse fragility curve obtained considering structural degradation with that of Figure 5 without degradation. In particular, the blue line represents the original non-damaged collapse fragility curve with data points (x), and the red line is the updated collapse fragility curve with data points (dots) caused by stiffness degradation during EQ19. There is notable change in risk, as expected, for this proof of concept analysis.

It can be seen that the collapse potential represented by the probability of collapse at discrete hazard levels increased as stiffness degradation with the assumed model in Equations 11-12 occurred. More importantly, the change of the collapse safety is also quantified over events due to the use of the HLA method, which is not the case for the static pushover methods typically used. In particular, the collapse capacity or IM in $10 \%$ probability of collapse after the degradation dropped to 4.9 from the original curve (Figure 5) value of 6.4, which can be compared to any designed tolerable probability of collapse such as $10 \%$ probability of collapse at the $2 \%$ probability in 50 years with $90 \%$ confidence [20]. It thus provides more intuitive and accurate estimates of potential earthquake induced losses, which enables improved decision-making for hazard mitigation. 


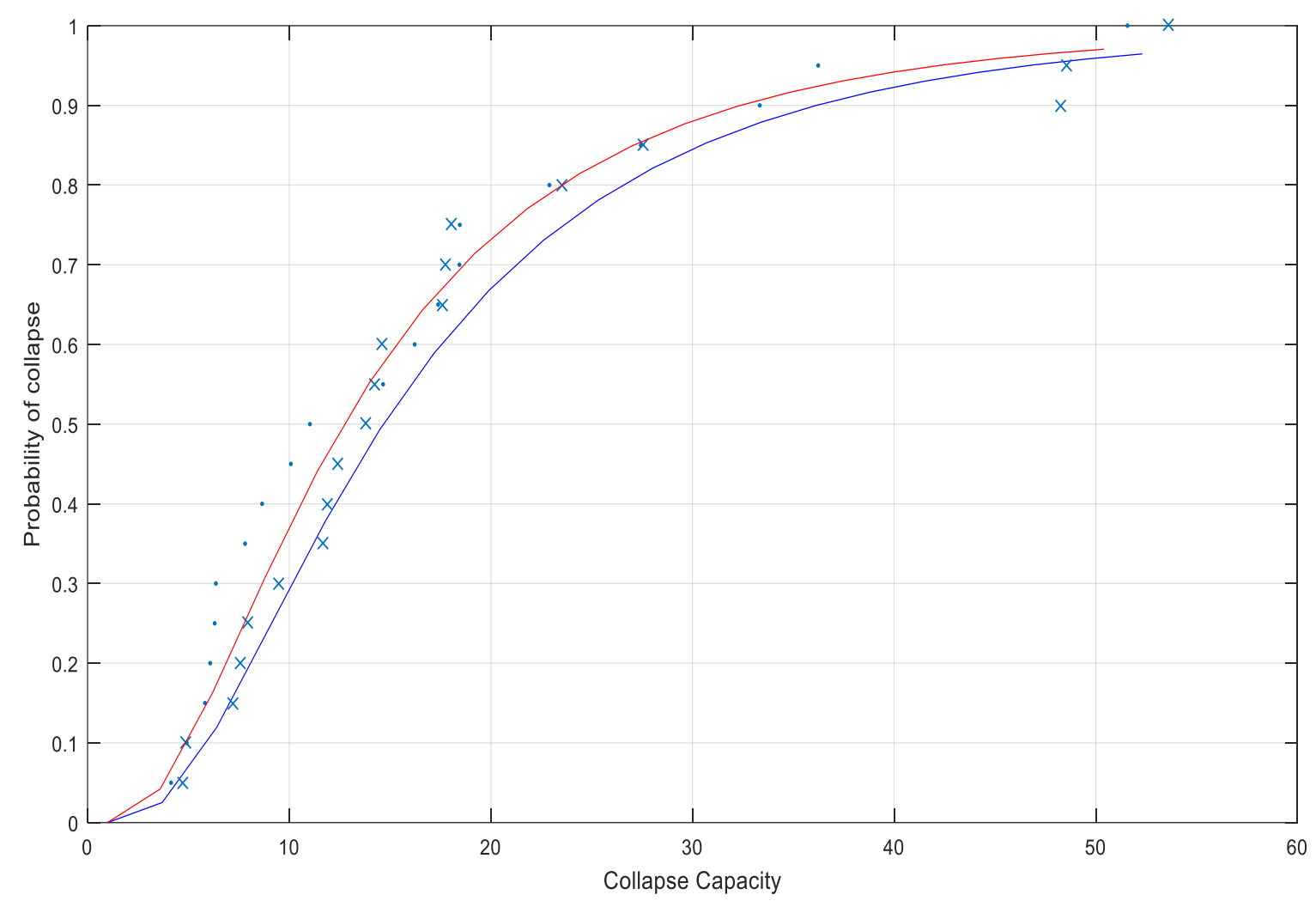

Figure 8. Comparison of collapse fragility curves before and after a significant earthquake event (EQ19), where the blue line represents the original non-damaged collapse fragility curve with data points (x), and the red line represents the updated collapse fragility curve with data points (dots).

This analysis clearly shows the combination of HLA and IDA analysis can provide the same results as a nonlinear analysis, validating the concept. The main advantage is the robustness implied by the ability of the HLA method to accurately identify structural parameters through a major event. Thus, there is no need to assume model parameters for an already damaged building for the IDA analysis, if the desired result is the current collapse capacity curve and consequent risk of collapse.

In addition, the combined HLA and IDA algorithms can be done within 15 minutes using Matlab on a 3.6GHz Intel Core i-7 machine. More importantly, the computational time could be significantly reduced to one minute or a few seconds by running different events in parallel with a more efficient $\mathrm{C} / \mathrm{C}+$ program or computer. Therefore, the overall combined method shows the potential to offer a 
rapid or effectively real time risk assessment of collapse probability and decision-making within a few minutes or less.

In terms of benefits and limitations, the results in Figure 8 also show the clear need for predictive structural models that can accurately predict potential degradation. In particular, while HLA SHM can identify current parameter values effectively, it cannot predict how the structure would degrade for a future event. In this analysis, the limitation is the assumed degradation model of Equations 11-12, where the plasticity defined in the bilinear model can be difficult to quantify the exact energy dissipation and degradation. While realistic, it may not hold for a given structure, impacting the benefit obtained from the resulting IDA and risk assessment when degradation is included. There is thus a need for what is effectively a "virtual structure" with predictive capacity for future events, but built off identified current values, similar to biomedical engineering "virtual patients" [41-44]. Thus, a more complex smooth hysteretic model, such as a Bouc-Wen model $[45,46]$ could be used to capture the continuous change of plasticity in the future work. However, this proof of concept analysis clearly shows how collapse risk can be accurately quantified if such predictive capability can be obtained, and thus shows a clear need for such a capability.

This study has some further limitations. In this case, the added noise has been shown to be larger than expected and the HLA method is robust [14, 25, 27], creating a conservative case analysis. However, if data were measured not at high enough resolution so only simple models could be identified, the method presented could be limited in the quality of assessment. Thus, as with any SHM or parameter identification method, sensor quality, location, and quantity directly relate to the quality of the identified parameters $[47,48]$. 
Equally, if the sensors had specific, unknown bias, or larger than expected errors or failure, the analysis would be flawed and the assessment inaccurate. There is thus the reduced risk of assuming a model and parameters for a damaged structure with this approach, where an inaccurate assumed model will impact the results [14]. However, this aspect needs further study and reinforces the need to both more extensively validate the results presented experimentally and/or in situ, as well as the need for better predictive nonlinear dynamic models developed from identified SHM results, which would extend SHM from a damage monitoring role into the foundation of prediction and risk assessment. 


\section{Conclusions}

This paper presents a novel combined SHM and IDA methodology to assess collapse risk for a structure after a major event, a major need in providing better post-event information for decision making on building occupation and the safety of surrounding areas. It also quantifies the increased risk when structures realistically degrade further under large post-event aftershocks, such as those seen in the Christchurch series of events in 2010-11. This latter result thus highlights the needs for not only implemented SHM to assess current capability, but also the ability to utilise these results in predictive models, which currently do not exist. However, the analysis presented clearly shows the potential of accurate assessment of collapse risk by combining SHM and nonlinear dynamic analysis methods. Importantly, the results were obtained with relatively very low computational effort, so the results would be available very soon post-event. Although this paper excluded strength degradation a similar method to the stiffness degradation could be used to account for cyclic deterioration of strength following seismic events, and is readily identified and thus accounted for by using the HLA method. Finally, the overall method is generalisable to assess the risk associated with any typical engineering demand parameter, as well as to thus utilise these methods with existing methodologies to extend them to financial risks and annualised estimated costs. 
Funding: No funding was received for this study.

Conflict of Interest: The authors declare that they have no conflict of interest.

\section{References:}

1. Rafaa Abbas, A.H., Stability Analysis of the Seismic Response of High Rise Steel Buildings including P-Delta Effects Applied Research Journal, 2016. 2(8): p. 353-361.

2. Adam, C. and C. Jager, Simplified collapse capacity assessment of earthquake excited regular frame structures vulnerable to P-delta. Engineering Structures, 2012. 44: p. 159-173.

3. Azarbakht, A. and M. Dolsek, Progressive Incremental Dynamic Analysis for First-Mode Dominated Structures. Journal of Structural Engineering-Asce, 2011. 137(3): p. 445-455.

4. Han, S.W. and A.K. Chopra, Approximate incremental dynamic analysis using the modal pushover analysis procedure. Earthquake Engineering \& Structural Dynamics, 2006. 35(15): p. 1853-1873.

5. Fenwick, R.C. and e. al, P-delta actions in seismic resistane structures Bulletin of the New Zealand National Society for Earthquake Engineering 1992. 25(1): p. 56-69.

6. Adam, C. and C. Jager, Seismic collapse capacity of basic inelastic structures vulnerable to the P-delta effect. Earthquake Engineering \& Structural Dynamics, 2012. 41(4): p. 775-793.

7. Tsantaki, S., L.F. Ibarra, and C. Adam, Effect of P-delta uncertainty on the seismic collapse capacity and its variability of single-degree-of freedom systems. Bulletin of Earthquake Engineering, 2015. 13(4): p. 1205-1225.

8. Effects of Strength and Stiffness Degradation on Seismic Response FEMA P440A 2009.

9. Di Sarno, L., Effects of multiple earthquakes on inelastic structural response. Engineering Structures, 2013. 56: p. 673-681.

10. Miranda, E. and J. Ruiz-Garcia, Influence of stiffness degradation on strength demands of structures built on soft soil sites. Engineering Structures, 2002. 24(10): p. 1271-1281.

11. Moghaddasi, M., et al., Seismic design of yielding structures on flexible foundations. Earthquake Engineering \& Structural Dynamics, 2015. 44(11): p. 1805-1821.

12. Moghaddasi, M., et al., Sensitivity analysis for soil-structure interaction phenomenon using stochastic approach. Journal of Earthquake Engineering, 2012. 16(7): p. 1055-1075.

13. Zhou, C., J.G. Chase, and G.W. Rodgers, Efficient hysteresis loop analysis-based damage identification of a reinforced concrete frame structure over multiple events. Journal of Civil Structural Health Monitoring, 2017: p. 1-16.

14. Zhou, C., et al., Comparing model-based adaptive LMS filters and a model-free hysteresis loop analysis method for structural health monitoring. Mechanical Systems and Signal Processing, 2017. 84: p. 384-398.

15. Clifton, C., et al., Steel structures damage from the Christchurch earthquake series of 2010 and 2011. Bulletin of the New Zealand Society for Earthquake Engineering, 2011. 44(4): p. 297-318.

16. Mander, J.B., et al., Incremental dynamic analysis applied to seismic financial risk assessment of bridges. Engineering Structures, 2007. 29(10): p. 2662-2672.

17. Gupta, A. and H. Krawinkler, Dynamic P-delta effects for flexible inelastic steel structures. Journal of Structural Engineering, 2000. 126(1): p. 145-154.

18. Vamvatsikos, D. and C.A. Cornell, Incremental dynamic analysis. Earthquake Engineering \& Structural Dynamics, 2002. 31(3): p. 491-514. 
19. Harrington, C.C. and A.B. Liel, Collapse assessment of moment frame buildings, considering vertical ground shaking. Earthquake Engineering \& Structural Dynamics, 2016. 45(15): p. 24752493.

20. Zareian, F. and H. Krawinkler, Assessment of probability of collapse and design for collapse safety. Earthquake Engineering \& Structural Dynamics, 2007. 36(13): p. 1901-1914.

21. Themelis, S., Pushover analysis for seismic assessment and design of structures. 2008, HeriotWatt University.

22. Adam, C. and C. Jäger, Simplified collapse capacity assessment of earthquake excited regular frame structures vulnerable to P-delta. Engineering Structures, 2012. 44: p. 159-173.

23. Xu, C., J.G. Chase, and G.W. Rodgers, Physical parameter identification of nonlinear baseisolated buildings using seismic response data. Computers \& Structures, 2014. 145(1): p. 4757.

24. Xu, C., J.G. Chase, and G.W. Rodgers, Nonlinear regression based health monitoring of hysteretic structures under seismic excitation. Shock and Vibration, 2015. 2015.

25. Zhou, C., et al., Physical Parameter Identification of Structural Systems with Hysteretic Pinching. Computer-Aided Civil and Infrastructure Engineering, 2015. 30(4): p. 247-262.

26. Zhou, C., et al., Overall damage identification of flag-shaped hysteresis systems under seismic excitation. Smart Structures and Systems, 2015. 16(1): p. 163-181.

27. Zhou, C., et al., Damage assessment by stiffness identification for a full-scale three-story steel moment resisting frame building subjected to a sequence of earthquake excitations. Bulletin of Earthquake Engineering, 2017: p. 1-20.

28. Aydınoğlu, M. and Y. Fahjan, A unified formulation of the piecewise exact method for inelastic seismic demand analysis including the P-delta effect. Earthquake engineering \& structural dynamics, 2003. 32(6): p. 871-890.

29. Chenouda, M. and A. Ayoub, Probabilistic collapse analysis of degrading multi degree of freedom structures under earthquake excitation. Engineering Structures, 2009. 31(12): p. 29092921.

30. Dhakal, R.P., J.B. Mander, and N. Mashiko, Identification of critical ground motions for seismic performance assessment of structures. Earthquake Engineering \& Structural Dynamics, 2006. 35(8): p. 989-1008.

31. Solberg, K.M., et al., Computational and rapid expected annual loss estimation methodologies for structures. Earthquake Engineering \& Structural Dynamics, 2008. 37(1): p. 81-101.

32. Asgarian, B., et al., INCREMENTAL DYNAMIC ANALYSIS OF HIGH-RISE TOWERS. Structural Design of Tall and Special Buildings, 2010. 19(8): p. 922-934.

33. Sommerville, P., et al., Development of Ground Motion Time Histories For Phase II Of The FEMA/SAC Steel Project, SAC Background Document Report SAC/BD-97/04. 1997.

34. Villaverde, R., Methods to assess the seismic collapse capacity of building structures: State of the art. Journal of Structural Engineering, 2007. 133(1): p. 57-66.

35. Teran-Gilmore, A., E. Avila, and G. Rangel, On the use of plastic energy to establish strength requirements in ductile structures. Engineering structures, 2003. 25(7): p. 965-980.

36. Teran-Gilmore, A. and J.O. Jirsa, A damage model for practical seismic design that accounts for low cycle fatigue. Earthquake Spectra, 2005. 21(3): p. 803-832.

37. Baber, T.T. and M.N. Noori, Random vibration of degrading, pinching systems. Journal of Engineering Mechanics, 1985. 111(8): p. 1010-1026.

38. Baber, T.T. and M.N. Noori, Modeling general hysteresis behavior and random vibration application. Journal of Vibration, Acoustics, Stress, and Reliability in Design, 1986. 108(4): p. 411-420. 
39. Christopoulos, C., A. Filiatrault, and B. Folz, Seismic response of self-centring hysteretic SDOF systems. Earthquake Engineering \& Structural Dynamics, 2002. 31(5): p. 1131-1150.

40. Limpert, E., W.A. Stahel, and M. Abbt, Log-normal Distributions across the Sciences: Keys and Clues: On the charms of statistics, and how mechanical models resembling gambling machines offer a link to a handy way to characterize log-normal distributions, which can provide deeper insight into variability and probability-normal or log-normal: That is the question. AIBS Bulletin, 2001. 51(5): p. 341-352.

41. Chase, J.G., et al., Validation of a model-based virtual trials method for tight glycemic control in intensive care. Biomed Eng Online, 2010. 9: p. 84.

42. Chase, J., T. Desaive, and J.C. Preiser, Virtual Patients and Virtual Cohorts: A New Way to Think About the Design and Implementation of Personalized ICU Treatments, in Annual Update in Intensive Care and Emergency Medicine, J.L. Vincent, Editor. 2016. p. 435-448.

43. Chase, J.G., et al., Next-generation, personalised, model-based critical care medicine: a stateof-the art review of in silico virtual patient models, methods, and cohorts, and how to validation them. Biomed Eng Online, 2018. 17(1): p. 24.

44. Dickson, J.L., et al., Generalisability of a Virtual Trials Method for Glycaemic Control in Intensive Care. IEEE Trans Biomed Eng, 2017.

45. Bouc, R., Forced vibration of mechanical systems with hysteresis, in Proceedings of the fourth conference on non-linear oscillation. 1967: Prague, Czechoslovakia.

46. Wen, Y.-K., Method for random vibration of hysteretic systems. Journal of the engineering mechanics division, 1976. 102(2): p. 249-263.

47. Docherty, P.D., et al., A graphical method for practical and informative identifiability analyses of physiological models: A case study of insulin kinetics and sensitivity. Biomedical Engineering Online, 2011. 10(1): p. 1-20.

48. Ljung, L., System identification : theory for the user. 2nd ed. Prentice Hall information and system sciences series. 1999, Upper Saddle River, NJ: Prentice Hall PTR. xxii, 609 p. 